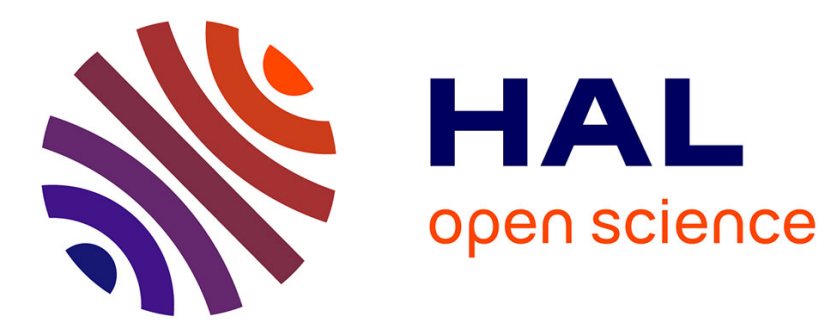

\title{
Knowledge-based systems for automatic ventilatory management.
}

\author{
Michel Dojat, Laurent J. Brochard
}

\section{To cite this version:}

Michel Dojat, Laurent J. Brochard. Knowledge-based systems for automatic ventilatory management.. Respiratory Care Clinics, 2001, 7 (3), pp.379-96, viii. inserm-00402401

\section{HAL Id: inserm-00402401 https://www.hal.inserm.fr/inserm-00402401}

Submitted on 7 Jul 2009

HAL is a multi-disciplinary open access archive for the deposit and dissemination of scientific research documents, whether they are published or not. The documents may come from teaching and research institutions in France or abroad, or from public or private research centers.
L'archive ouverte pluridisciplinaire HAL, est destinée au dépôt et à la diffusion de documents scientifiques de niveau recherche, publiés ou non, émanant des établissements d'enseignement et de recherche français ou étrangers, des laboratoires publics ou privés. 


\section{Knowledge-Based Systems for Automatic Ventilatory Management}

Michel Dojat, Eng, $\mathrm{PhD}^{*}$, Laurent Brochard, $\mathrm{MD}^{+}$

"Research Engineer, Institut National de la Santé et de la Recherche Médicale, INSERM U492, Créteil, France.

${ }^{+}$Professor of Intensive Care Medicine, Service de Réanimation Médicale, Hôpital Henri Mondor, AP-HP, Créteil, France.

To appear in: G. Iotti, eds., Respiratory Care Clinics. Special Issue on Closed-Loop Control Mechanical Ventilation. W.B. Saunders Co., Philadelphia, 2001 (june) Note: This is a preprint and may differ in minor details from the published version

Corresponding author for proof and reprints:

Michel Dojat, Eng, PhD

INSERM U438

CHU de Grenoble - Pavillon B

BP 217

F-38043 Grenoble Cedex 09, France

(33) 476765748

(33) 476765896 (fax)

e-mail:mdojat@ujf-grenoble.fr
Prof. Laurent Brochard MD

Medical Intensive Care Unit

Hôpital Henri Mondor

51, avenue de Maréchal de

Lattre de Tassigny

F-94010 Créteil, France

(33) 149812545

(33) 142079943 (fax)

e-mail : laurent.brochard@hmn.ap-hopparis.fr 


\section{Introduction}

Despite their enormous potential to facilitate bedside management, the practical role of computers in critical care environments is generally restricted to the storage and the retrieval of data coming from electronic medical devices and hospital information networks. Benefits of the use of computers in health care may be extended by the design of computerized medical assistants that can efficiently discharge the clinical staff of repetitive tasks (which, in practice, often are not performed) and, importantly, help practitioners to make efficient decisions in time. In intensive care and anesthesia, the demand for computerized medical assistants is potentially considerable, in order to filter and synthesize the growing mass of clinical parameters and information available. The progressive introduction of computerized protocols has been proposed to standardize the bedside decision making process for mechanical ventilation and to reduce unnecessary variation among practitioners ${ }^{32}$, reinforcing the potential impact of computerized medical assistants. Designing such assistants for intelligent monitoring, diagnosis and therapy planning tasks in Intensive Care and Anesthesia is a challenging goal that requires the modeling of several levels of knowledge ranging from low level data interpretation to high level cognitive tasks, such as planning (for a review of recent research work in this field see ${ }^{12,25}$ ). Our goal in this special issue is to show how knowledge-based computerized assistants can be used to practically improve the closed-loop control of mechanical ventilation.

\section{Control and Planning: Two Key Points for Automatic Ventilation}

\section{Management}


Modern methods of mechanical ventilation partially assist the patient's ventilation by adding a variable amount of mechanical support to his/her spontaneous activity. In this context, since the needs of the patient are evolutive, it is essential to continuously control the ventilatory support, in order to avoid excessive work of breathing and effort, discomfort and dyspnea on the one hand, or excessive support, hyperinflation and dyssynchrony on the other hand. In parallel to this ideal automatic adaptation, it may be necessary to plan the long term adaptation of the therapy according to specific medical goals. For instance, it may be indicated to gradually decrease the level of assistance in order to facilitate the weaning from the ventilator or to take into account large variations of physiological needs during the patient wake-up from anesthesia or drug intoxication.

Planning and control are two different tasks that have a common goal: choosing actions over time to influence a process, based on some model of that process $^{9}$. Control is a local task to determine what to do the next instant. Planning is a strategic task to regulate the process evolution. For control and planning, numerous techniques are available, respectively coming from two disciplines: Control Theory and Artificial Intelligence (AI), which differ mainly in the types of process models used. Control and planning are two complementary and essential tasks that must be combined to design multi-level controllers for the automatic supervision of complex systems such as mechanical ventilation of patients.

\section{Basic Control Loops in Mechanical Ventilation}

In the field of mechanical ventilation we can identify three levels of control (L1, L2 and L3) $)^{14}$ (see Fig. 1). The complexity and the response time of the levels increase from the lowest to the highest level of control. Each level controls the levels below and is in turn controlled by the levels above: 


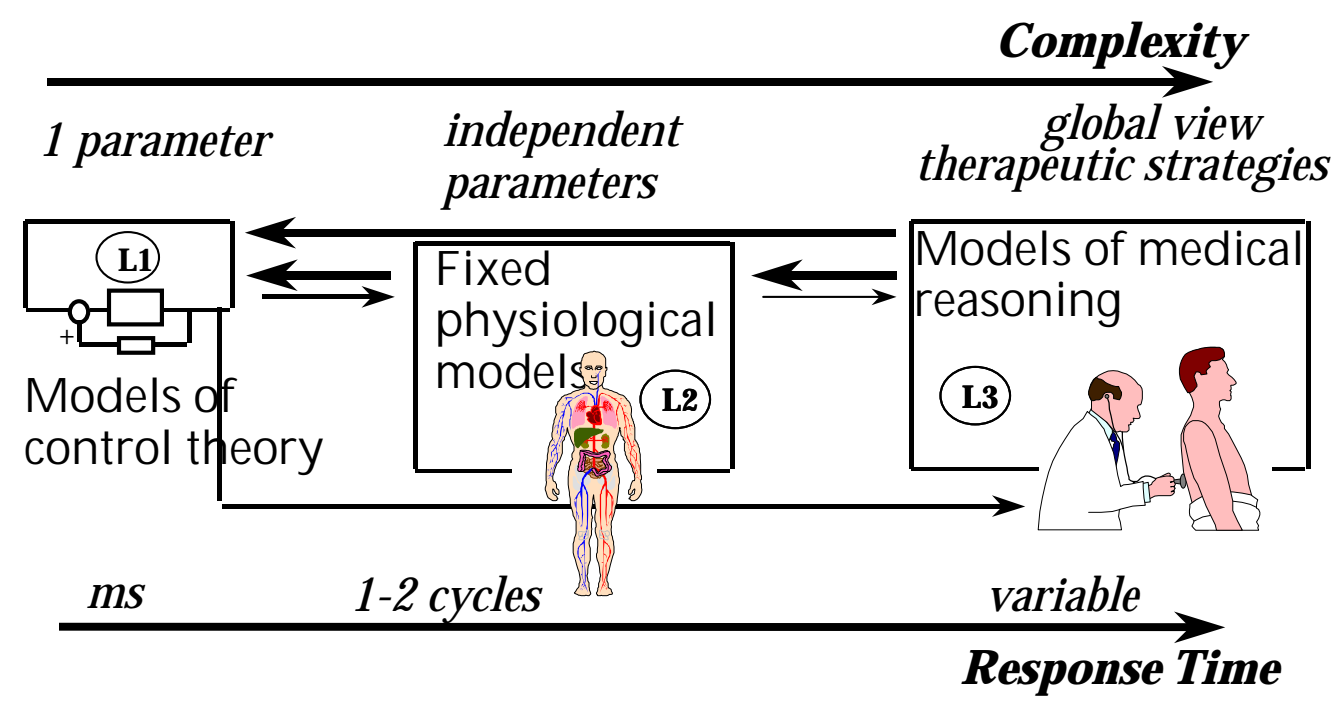

Figure 1: Different levels of control (Reprinted from Artif Intell in Med, 11, Dojat M, Pachet F, Guessoum Z, et al, NeoGanesh: A Working System for the Automated Control of Assisted ventilation in ICUs, 97-117, Copyright (1997), with permission from Elsevier Science).

Thick arrows indicate the control from the highest levels over the lowest levels. Thin arrows indicate the information flux (alarms, acknowledgments, ...) which goes through the hierarchy.

- L1 is a highly reactive basic control loop of the ventilator (response time $\approx 1 \mathrm{~ms}$.) that controls essentially the flow or the pressure sent to the patient by driving a servo-valve. This can be used in complex modes like proportional assist ventilation (PAV).

- L2 determines the mode of ventilation and has been used for minute mandatory ventilation (MMV) for instance. The response time of L2 is approximately of 1 or 2 cycles (few seconds).

- L3 represents the adaptation of the mechanical assistance using information about the current state of the patient, its evolution and the predefined therapeutic goals. This level of control, relying on specific medical knowledge and therapeutic strategies, is traditionally realized by the clinician in charge. The response time at this level varies from a few seconds in alarming situations to a few minutes in routine patient observation. 
L1 and L2 may be directly assimilated to low-level control or tactical component that continuously adjusts the mechanical assistance in function of the patient's state evolution, and L3 to high-level planning or strategic component that influences the behavior of the tactical component in order to follow specific therapeutic plans ${ }^{9}$.

\section{Low-Level Controllers}

Based on control theory we can design a component which, starting from direct observations of the system behavior (measured variables), estimates its current state and regulates it by performing actions (on set variables) to reach a target state (the set-point). L1 and L2 are designed using such a standard principle. To estimate and to regulate, L1 relies on mathematical models of the physical components of the ventilator and L2 on a physiological model of the patient based on equations of ventilatory mechanics. For L3, several sophisticated automatic controllers have been proposed, some of them are detailed in this special issue ${ }^{3}$, and are implemented in ventilators. To compensate their lack of prediction, these controllers react as quickly as possible to any change between the current state and the target state. They are efficient, robust and rely on well-mastered techniques of control theory. However, their view of the patient is restricted to the model they are based on. Therefore, the performance of these automatic controllers may be poor, when they are applied to various pathologies and emergency situations where the underlying assumptions of the model are generally no more valid. For instance, parameters considered as constant in the model may change over time with the disease evolution. The algorithms they are based on can not reflect the attitude of the physician who dynamically adapts the strategy over time, based on the 
evolution of patient's state and therapeutic goals. The integration of heuristics such as "never envisage full weaning at night" is difficult.

In order to perform a diagnosis, determine a therapy and act on the ventilator, a substantial part of L3 might be integrated into the overall system. An attempt to integrate into the controller an active clinical strategy, represented with production rules (IF conditions THEN actions), has been proposed by Strickland and Hasson ${ }^{46,47}$. Their system used two parameters, tidal volume (Vt) and respiratory frequency (RR), to estimate the current patient's ventilation and modify, if necessary, the mandatory frequency and the pressure support level (the SIMV with Pressure Support mode was used). Oxygen saturation was used as a safety parameter. When ventilation was judged correct $(\mathrm{Vt} \geq 5 \mathrm{ml} / \mathrm{kg}$ and $8 \leq \mathrm{RR} \leq 30$ cycles/min), mandatory frequency and pressure support level were systematically decreased. The clinical results indicated that the use of the system reduced the time spent with an incorrect ventilation and the number of blood gas measurements performed. In this system, the weaning strategy was reactive, fixed and did not take into account the temporal evolution of ventilation, a central point in the clinician's decision making process. Consequently, only candidates for weaning were ventilated with this system.

The work performed since several years at the LDS Hospital for the elaboration of computerized protocols is relevant here ${ }^{17,32,37}$. The algorithmic-oriented approach chosen at the LDS Hospital leads to a complex logic where temporal aspects are intricate. It is largely recognized in knowledge acquisition community that the elicitation of the knowledge level ${ }^{36}$ (such as for instance temporal abstractions ${ }^{42}$ applied to the context of the management of mechanical ventilation) is essential to facilitate reuse, sharing and maintenance of knowledge-based systems $^{34}$. Recently, the need to base automatic control on clinical experience rather than on mathematical models of the couple patient-ventilator has led to the introduction of fuzzy logic in working closed-loop ${ }^{41}$ or open-loop ${ }^{35}$ systems. Fuzzy logic is used to represent the subjective human notions employed in decision-making, such as "high", "low", "normal" or "too high". Rules, sets and membership functions (the central elements of the fuzzy logic approach) are 
designed according to medical practice. Promising results were obtained using this type of fuzzy controller for automatic pressure support level adjustment ${ }^{35}$. Here again, the introduction of (fuzzy) temporal reasoning is essential to envisage the automation of the entire mechanical ventilation process. Indeed, integrating L3 in the system should allow the construction of a more comprehensive view of the time course of the patient's state, thereby providing it the ability to manage several ventilation strategies depending on the patient's state. The results we have recently obtained at Henri Mondor Hospital (Créteil, France), demonstrate that this is feasible and that a knowledge-based approach is suitable to model a substantial part of the clinician's expertise relevant for L3.

\section{Knowledge-Based Systems for Ventilation Management}

Standard systems for patient monitoring are built with three functions: acquisition, storage and visualization of medical data to facilitate their management by the clinical staff. Knowledgebased systems applied to this context (see Figure 2) are enriched with two main modules, the scenario recognition module that recognizes the current situation as it is developing, based on past and present information available, and the action planning module that defines the best therapeutic actions to be performed and their expected effects for restoring acceptable physiological conditions. The envisaged actions plan can be proposed to the user (open-loop system) or directly performed by the computerized system (closed-loop system). A minority of medical systems work currently in closed-loop. For scenario recognition and decision making, knowledge-based systems can rely on an explicit model either of the patient or of the medical expertise required to perform a task. 


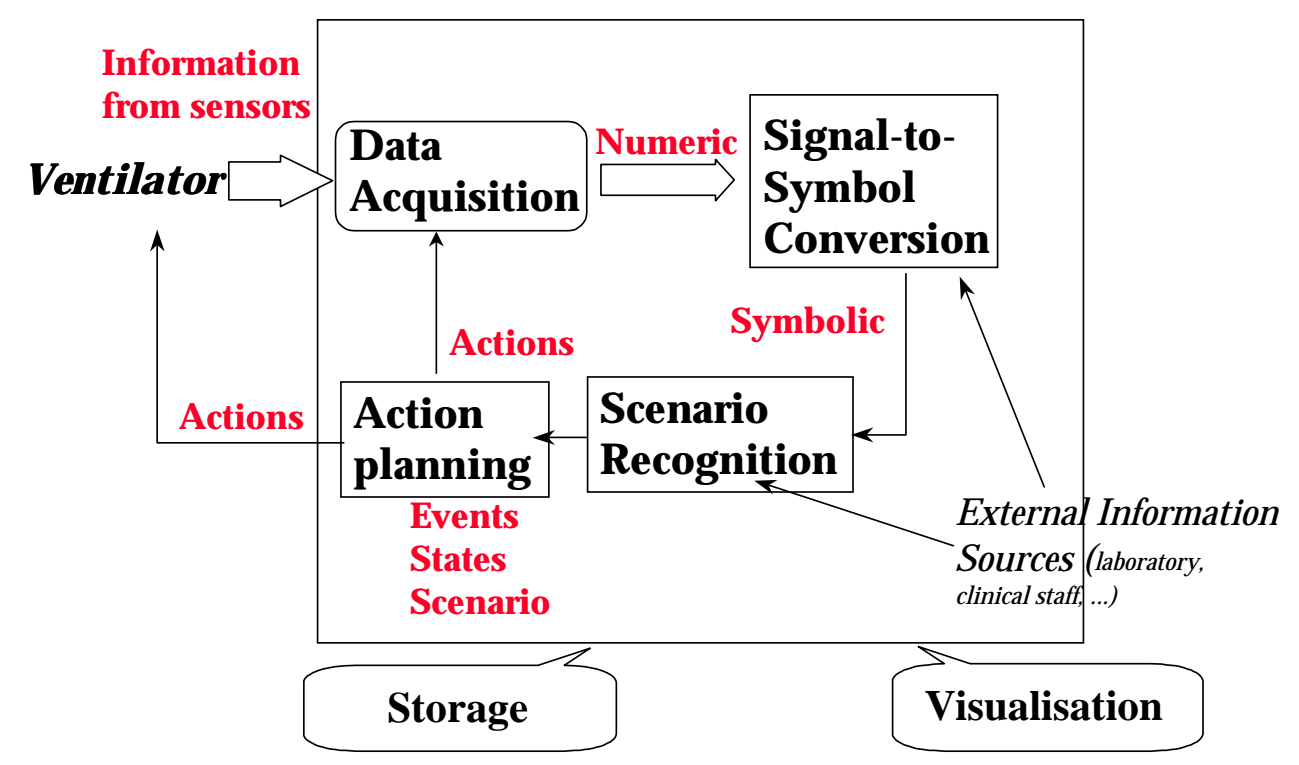

Figure 2: Architecture of a knowledge-based system for ventilation management (Adapted from Artif Intell in Med, 14, Dojat M, Ramaux N, and Fontaine D, Scenario Recognition for Temporal Reasoning in Medical Domains, 139-155, Copyright (1998), with permission from Elsevier Science).

Each rectangle represents a specific step. After data acquisition step, numeric data are transformed to symbolic values (signal-to-symbol conversion) used to described the process (classification of the ventilation, qualitative trends, stability, ...). After recognition of the situation (scenario recognition), therapeutic actions (action planning) are performed on the ventilator in case of a closed-loop system. Modifications of the data acquisition conditions for instance, sample frequency rate or acquisition of additional parameters, can be realized.

Planning consists in the explicit definition of the actions sequencing to be executed in a given context to reach the goal. For ventilation management the general plan showed in Figure $3 \mathrm{a}$ can be used. After data acquisition, the ventilation is classified and the current patient's state is determined. Based on the history of patient's ventilation, temporal reasoning allows to assess the evolution of the patient's state. The therapy prescription implies the evaluation of the adequacy of the current therapy, function of its expected effects and its evolution. This evaluation allows for the definition of the actions to perform and of the new state to expect. The decomposition of each 
task is realized up to the description of simple actions to be executed. This general plan is instantiated for specific situations as shown in the example in Figure $3 b$.
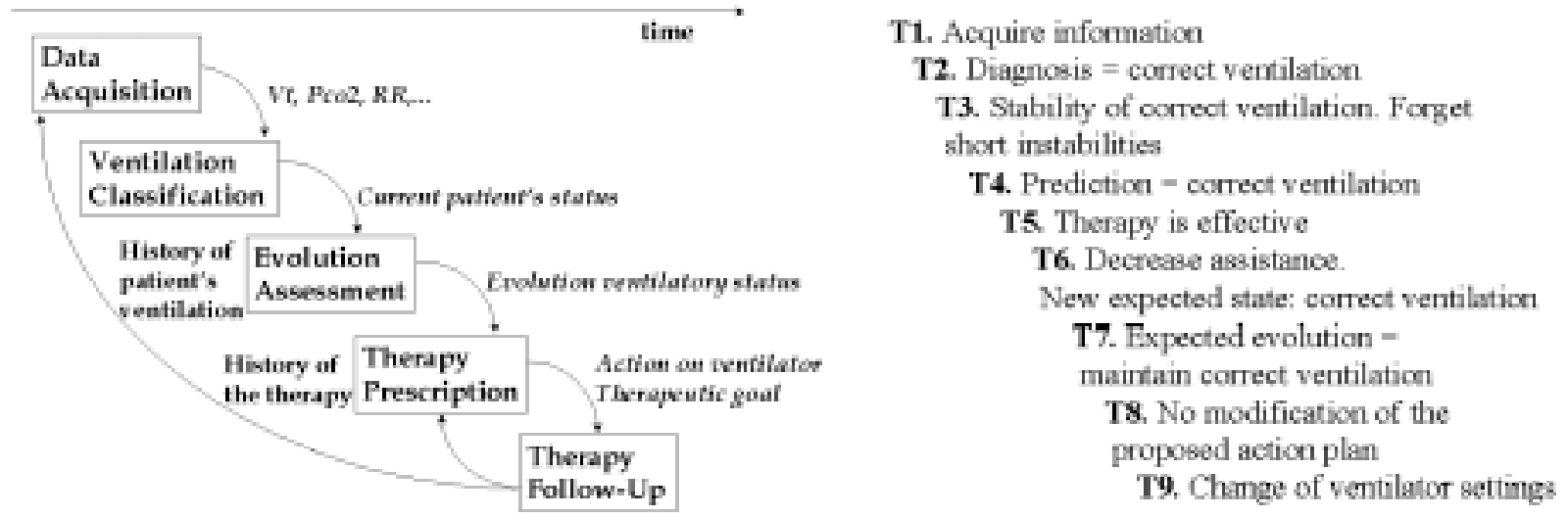

Fig. 3a-b: Plans for ventilation management

3a (Left): The general plan for the set of steps during ventilation management. The steps are ordered but the sequencing is interrupted in case of alarming situation.

3b (Right): This figure represents the instantiation of the general plan for a specific situation: the restoration of correct ventilation after a short episode of tachypnea. After observation of the patient's ventilation (T1), correct ventilation is confirmed (T2). The ventilation is globally correct and the short episode of tachypnea is eliminated (T3). Persistence of correct ventilation is predicted (T4) and then the current therapy is adapted (T5). Mechanical assistance has been increased to combat the tachypnea episode. The replacement of the low initial level of pressure support is proposed (T6). The maintain of correct ventilation is expected (T7). The global plan is not modified (T8) and the ventilator settings is modified.

They are three essential aspects in knowledge-based systems applied to patient monitoring that are addressed by specific research efforts in artificial intelligence:

- Temporal Reasoning: starting from time-stamped physiological raw data, the system has to built several abstraction levels in order to assess the global evolution of the controlled process (i.e. the couple patient-ventilator). Temporal abstraction mechanisms have been proposed to tackle this problem ${ }^{15,22,42,45}$. Temporal aspect has to be handled also at the acquisition level to 
assure the quality of the data and the distinction of significant events from artifacts ${ }^{4,24}$. The system should be able to maintain (in closed-loop) the patient into a zone of respiratory comfort and to react in time to alarming situations. Ideally, the amount of time to take a decision must be known in advance to judge of its adequacy to the situation. Specific mechanisms for the preemption of decisions at any time are required to respect real-time constraints $^{51}$.

- Reusing knowledge: elicitation, validation and maintenance of knowledge-rich clinical decision-support systems are laborious efforts. Several research works are performed to facilitate, via the design of specific knowledge acquisition tools $\mathrm{s}^{33,43}$ or of standardized medical ontologies $^{7,38}$, the sharing, reuse and maintenance of knowledge bases.

- Distributed components: Automatic supervision of patient is a complex task that implies solving different kind of problems. Distributed architectures, where several heterogeneous specialized modules cooperate, can help to partition problems and implement efficient systems integrated into the clinical environment ${ }^{21,26}$.

\section{Current State of the Art}

A few knowledge-based systems for automatic management of mechanical ventilation have already been proposed. Despite several interesting results, this application remains a challenging task for researchers in medical AI, whereas there is a real clinical need for such systems. A recent review of different systems can be found ${ }^{18}$. We would like here to stress the major contribution of some selected systems that can help to design the next generation of medical assistants for mechanical ventilation. The crucial importance of time and context to interpret physiological raw data and to propose a therapy has been emphasized in the precursor system Ventilator Manager 
$(\mathrm{VM})^{20}$. The integration of the system into the hospital information network is a prerequisite for its acceptation by the clinical staff. $\operatorname{COMPASS}^{44}$ was based on a distributed blackboard architecture and connected to the information network HELP at the LDS Hospital. Besides the modeling of the clinician's expertise with production rules, KUSIVAR ${ }^{39}$ integrated a mathematical model of the couple patient-ventilator to simulate the effects of ventilator setting's modification on the physiological data. The potential interest of fuzzy logic to classify physiological parameters was firstly introduced in $\mathrm{VRM}^{1}$. The notion of advisor that criticizes the settings proposed by the clinician in charge was introduced with the VQ-ATTENDING system ${ }^{30}$. Such computerized advisors could be useful for the supervision of difficult patients such as those suffering from ARDS, or to control the compliance to computerized protocols or guidelines ${ }^{43}$. Elicitation of knowledge is a well-known difficult problem. RESPAID ${ }^{6}$ was the first attempt to use machine learning techniques (ID3 algorithm) to automatically create, from a set of examples, production rules linking physiological raw data to clinical events. The resulting set of rules was able to classify correctly alarming events in the remaining data set. Recent systems confirm the clinical interest of such an approach ${ }^{31,48}$.

The main characteristic of this first generation of systems is the difficulty to use them outside the laboratory where they have been built. At the opposite, recent systems are open architectures applied to intelligent patient monitoring, generally connected to the hospital network and integrating original techniques that can be used in other contexts ${ }^{40,23,27}$. The current AIDIAG project at Lille University Hospital (France) ${ }^{4,49}$, aims to collect, process, synthesize and interpret, using knowledge bases, the set of information available at bedside. The SIMON architecture ${ }^{8}$ is an extensible clinical platform to support clinical decision-making over the course of patient care, 
and operates in the Vanderbilt University Medical Center. The construction of knowledge bases to be incorporated into these two architectures is under development.

\section{A Practical and Successful Experience Using the NéoGanesh System}

The initial objective of the design of the knowledge-based system called NéoGanesh, was to build a closed-loop system 1) efficient for the automatic control of mechanical support, 2) which could be extended to gradually improve its reasoning and planning capabilities and 3) which could be tested at the patient's bedside to measure its performance at each step.

\section{A Knowledge-Based System Working in Closed-Loop}

Instead of computerizing a specific recipe for ventilation management ${ }^{37,47}$, in designing NéoGanesh we tried to respect the golden rules of knowledge engineering: make an explicit model of medical tasks and reasoning involved, and distinguish between the conceptual model (knowledge level, see for instance Figure 3) and the representation paradigms (symbolic level) used to implement $i t^{13}$. NéoGanesh is based on current AI techniques: a knowledge representation that mixes objects, rules and temporal abstractions ${ }^{16}$ in a distributed architecture ${ }^{14}$. It combines a "tactical" component and a "strategic" component. The "strategic" component relies on the model and representation of the intensivist's decision-making process. The "tactical" component uses three physiological parameters to modify the level of assistance during pressure support mode ventilation, and to maintain the patient within a zone of acceptable ventilation defined as Zone of Respiratory Comfort: $12<\mathrm{RR}<28$ cycles/min, $\mathrm{Vt}>300 \mathrm{ml}$ or 250 if weight $<55 \mathrm{Kg}, \mathrm{PetCO}_{2}<55$ $\mathrm{mmHg}$ or $65 \mathrm{mmHg}$ if $\mathrm{COPD})$. Therefore, compared to general architectures such as VENTPLAN $^{40}$ or GUARDIAN ${ }^{23,27}$, the approach is more modest. The originality of NéoGanesh can be stressed in three points: 
- Our system is based on the modeling of the medical expertise required to mechanically ventilate patients with the pressure support ventilation mode. It does not include mathematical equations of a physiological model. There are three reasons for that: i) in pathological situations, physiological models are uncertain and can require data that are not available in real-time, or data whose the estimation is difficult or imprecise. Data validation is still an open problem; ii) physiological models do not always represent useful information to the clinician in decision making. For instance, to follow up the recovery of patient after anaesthesia, pharmacological equations are imprecise and not used in practice; iii) the decision making process of clinicians may be less variable than the complex physiology of patients. This is reinforced by the introduction of protocols or guidelines for mechanical ventilation based on objective measurements like respiratory frequency or the rapid shallow breathing index. In conclusion, it seems simpler to model decision-making based on objective measurements, rather than based on physiology and multiple assumptions of the patient's behavior. Therefore the NéoGanesh system is more a "decision-driven" system than a "patient-driven" system, although it indeed uses data coming from the patient.

- The introduction of a new mode of ventilation such as $\mathrm{PAV}^{50}, \mathrm{ALV}^{28}$ or $\mathrm{ARIS}^{5}$ is a long and difficult process. Therefore, we chose i) to ventilate patients with a standard ventilation mode, pressure support ventilation, largely used for weaning, and ii) to add heuristic knowledge to improve its use and to facilitate the weaning process.

- It is essential to build extensible architectures for patient monitoring and implementation of mechanical ventilation protocols. Most of the recent systems proposed are very ambitious and their validation is only possible through simulators ${ }^{27}$. Our approach is more progressive. Starting from an extensible architecture, we integrated modules that are validated through clinical trials. 
NéoGanesh is based on a pragmatic bottom-up approach guided by a tight collaboration with intensivists and a continuous clinical evaluation. Our current module is devoted to the closedloop control of pressure support ventilation and decision for extubation. Our approach is closed to the VIE-VENT project at Vienna (Austria) ${ }^{24}$, that is devoted to the development of a knowledgebased system for mechanically ventilated newborn infants to optimize therapy planning and to support neonatologists in their daily routine. With VIE-VENT, a change of the ventilator's settings is evaluated by monitoring the trend of the subsequent changes of the transcutaneous blood gases. A new recommendation is formulated if the short-term trend does not meet present requirements concerning the direction and the amount of the expected change ${ }^{29}$. Therapy recommendations, based on transcutaneously and invasively determined blood gas measurements, are formulated in terms of recommended changes of the ventilator settings.

\section{Some Clinical Results}

NéoGanesh has been used in closed-loop and tested in more than sixty ventilated patients at Henri Mondor hospital (Créteil, France). We performed two types of evaluation i) one set of tests to assess the capacity of the system to control the level of assistance in accordance to the patient's needs (evaluation of the tactical level) and ii) a second set of tests to assess the decision of extubation provided by the system (evaluation of the strategic level).

\section{Evaluation of the Management of Mechanical Ventilation}

In a preliminary study, we have ventilated two different groups of patients, both with NéoGanesh. The two groups represented two different steps in the course of mechanical ventilation. The first group ( $\mathrm{n}=9$ ) was composed of patients considered as candidates for weaning, and the second one $(n=10)$ of severe patients needing to be maintained under mechanical ventilation. The mean time 


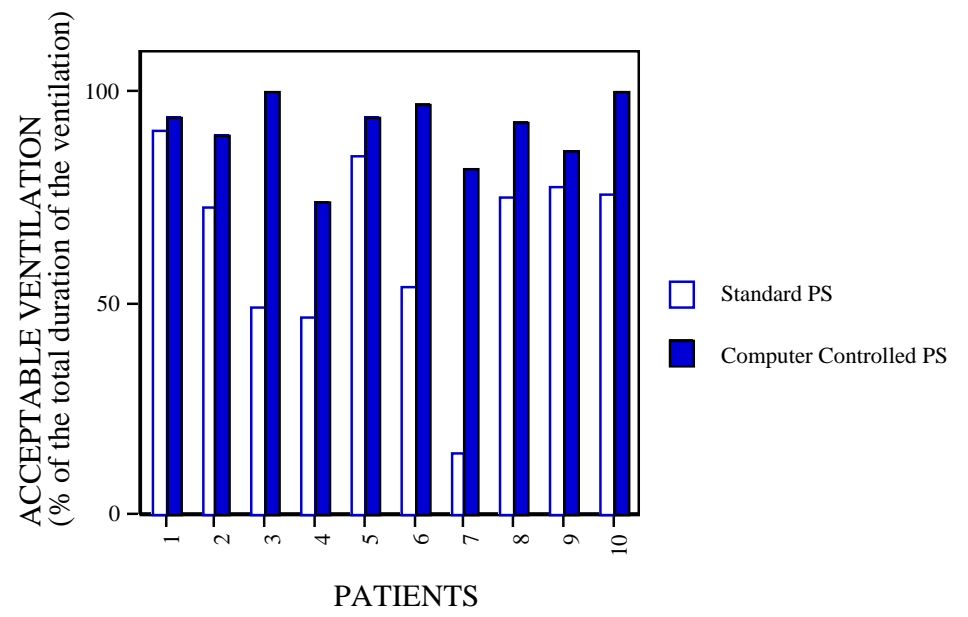

Figure 4: Mean time spent in the zone of respiratory comfort without (standard PS) and with NéoGanesh (Computer Controlled PS) expressed as the percentage of the total ventilation duration.

spent within the Zone of Respiratory Comfort (see above) expressed as the percentage of the total ventilation duration was $99 \%$ for the first group and $90 \%$ for the second group (see details $\mathrm{in}^{10}$ ).

In a more recent study, we ventilated randomly 10 patients $24 \pm 4$ hours with NéoGanesh and $23 \pm 3$ hours without standard pressure support ventilation (PSV) without NéoGanesh. In standard PSV, the clinician in charge could modify the pressure support level at his/her discretion. The mean pressure support level was similar with the two modes $\left(17 \pm 4 \mathrm{cmH}_{2} \mathrm{O}\right.$ and $19 \pm 6 \mathrm{~cm} \mathrm{H}_{2} \mathrm{O}$ without and with NéoGanesh, respectively). The mean time spent into the Zone of Respiratory Comfort was $66 \pm 24 \%$ and $93 \pm 8 \%$ without and with NéoGanesh, respectively. These results are shown in Figure 4. 


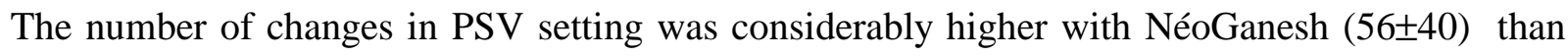
with standard PSV (1 \pm 2$)$. The mean time spent in a condition of critical ventilation $(R R>35$ cycles/min, $\mathrm{Vt}<300 \mathrm{ml}$ or PetCO2 $\geq 55 \mathrm{mmHg}$ ) was $3 \%$ with NéoGanesh compared to $23 \%$ with standard standard PSV. Lastly, the time spent with a high level of occlusion pressure (P0.1), suggesting a high work of breathing, was significantly reduced with the knowledge-based system. NéoGanesh tries to automatically decrease the level of pressure support. This is illustrated for the patient in Figure 5A, where the pressure support level was modulated, depending on the respiratory rate values, when using NéoGanesh, while it remained constant when using standard PSV. The patient shown in Figure 5B was hyper-assisted (RR $<12$ cycles/min) during $49 \%$ of total ventilation time with standard PSV. With NéoGanesh this situation never happened. When such a condition of hyper-assistance is detected, the pressure support level is automatically decreased by NéoGanesh.
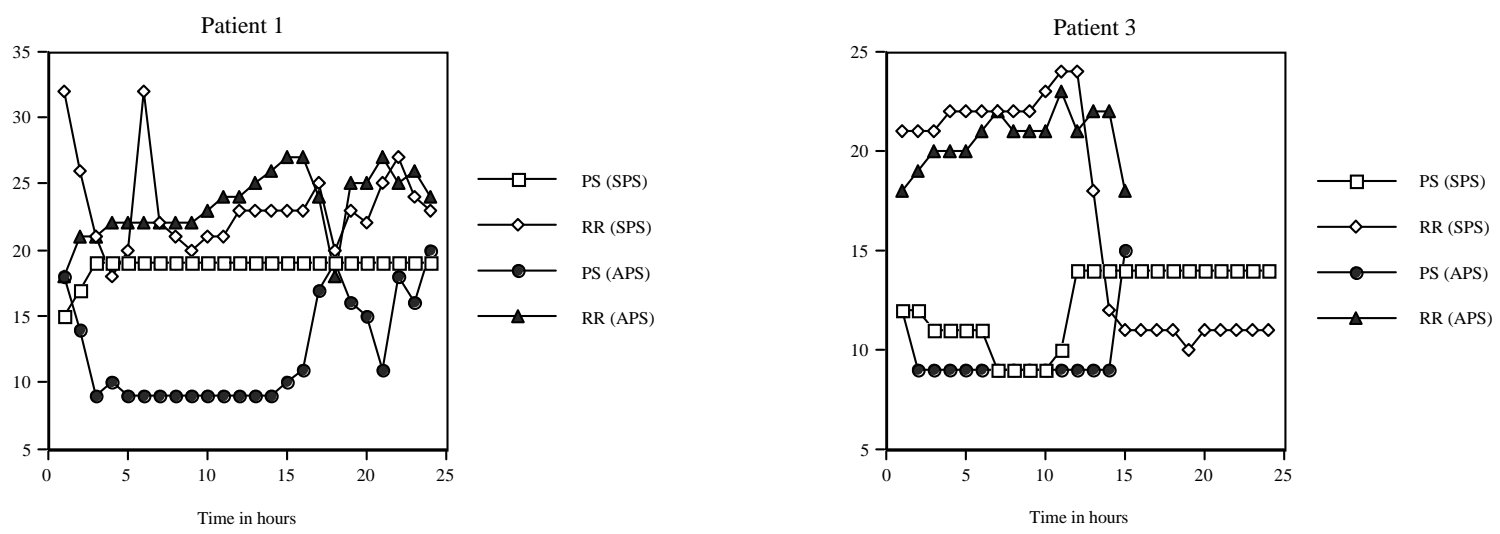

Figure 5: Evolution of pressure support level (PS) in $\mathrm{cmH}_{2} \mathrm{O}$ and respiratory rate (RR) in cycles/min with NéoGanesh (APS), and with standard pressure support ventilation without NéoGanesh (SPS). 
5A (Left): Results for patient 1 5B (Right): Results for patient 3

As indicated in Figure 6A, frequent episodes of transient tachypnea were avoided for patient 8 when using NéoGanesh. The price to pay was an increase of the mean pressure support level (17 $\mathrm{cmH}_{2} \mathrm{O}$ for standard PSV compared to $24 \mathrm{cmH}_{2} \mathrm{O}$ with NéoGanesh). The patient shown in Figure $6 \mathrm{~B}$ spent $54 \%$ of total time of ventilation in the Zone of Comfort for Ventilation when ventilated with standard PSV, and $97 \%$ of when ventilated with NéoGanesh. Again for this patient, NéoGanesh avoided episodes of tachypnea and contributed to the reduction of the rapid shallow breathing index $(\mathrm{RR} / \mathrm{Vt})$. Finally, the time spent with an estimated $\mathrm{P} 0.1>4 \mathrm{cmH}_{2} \mathrm{O}$ was lower with NéoGanesh than with standard PSV.
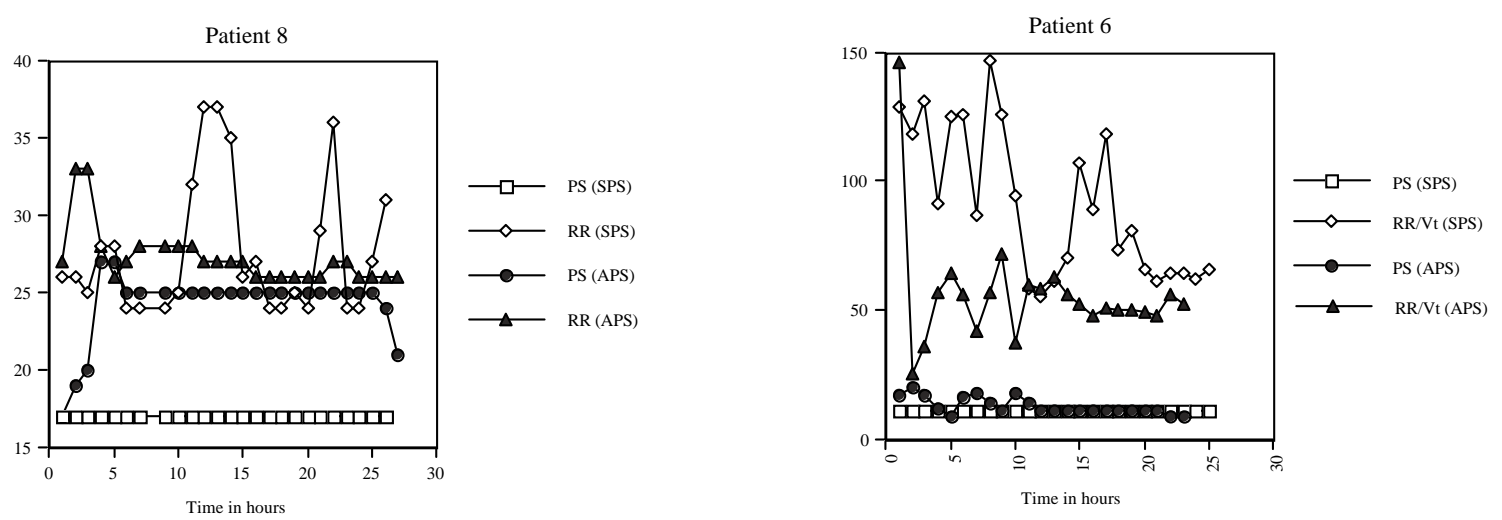

Figure 6: Evolution of pressure support level (PS) in $\mathrm{cmH}_{2} \mathrm{O}$ and respiratory rate (RR) in cycles/min or rapid shallow breathing index (RR/Vt) in cycles/min/l with NéoGanesh (APS), and with standard pressure support ventilation without NéoGanesh (SPS).

6A (Left): Results for patient 8 6B (Right): Results for patient 6Decision for Extubation 
For some patients weaning can be a long and difficult process. Continuous adjustment of mechanical assistance as performed by NéoGanesh may positively influence the weaning outcome. The level of pressure support may be a useful guide for determining the optimal time for performing tracheal extubation. This strategy was implemented in NéoGanesh: when the patient is ventilated with a low level of assistance $\left(9 \mathrm{cmH}_{2} \mathrm{O}\right.$ for patients with an endotracheal tube or $5 \mathrm{cmH}_{2} \mathrm{O}$ for patients with a tracheotomy cannula), an observation period is triggered (1 or 2 hours depending whether the level of pressure support after one hour of ventilation is 15 < or $\geq 15 \mathrm{cmH}_{2} \mathrm{O}$ respectively) and a decision about extubation is displayed on the computer screen. For 38 patients, we compared the decision given by NéoGanesh to the standard set of weaning tests (pre-weaning tests +2 hours on T-piece +48 hours of follow-up). The negative predictive value was equal in the two cases. However, the positive predictive value was of $89 \%$ for NéoGanesh and $77 \%$ for standard PSV, and $81 \%$ for the rapid shallow breathing index alone ${ }^{11}$. NéoGanesh predicted failure of weaning for 5 patients who tolerated the 2-hour T-piece trial but eventually failed weaning.

\section{Towards Smart Ventilators}

We have proposed to integrate medical knowledge into closed-loop controllers. Our clinical results indicate the potential interests of such an approach: adaptation of assistance to the needs of the patient, reduced need for monitoring and better weaning outcomes. Further studies should now be launched to demonstrate that this new technology improves patient care or that it maintains patient care while decreasing cost. Up to now, none of the sophisticated closed-loop controllers proposed in the literature have had a major impact on clinical care. One reason suggested $^{18}$ is that these systems are pure engineer-oriented products not related to common 
clinical practice. Clearly, in designing knowledge-based closed-loop controllers, we changed this view in adopting a clinician-oriented approach. Based on objective criteria, weaning protocols have been proposed by medical experts ${ }^{2,19}$. Results from a prospective multi-centre randomized clinical trial indicate that a computerized system for directing ventilator therapy can significantly improve morbidity ${ }^{17}$. We consider that, for ventilation management, medical knowledge is mature enough to be incorporated into smart ventilators that can really assist clinicians in bedside care. Our work with the NéoGanesh system constitutes a first step towards the construction of such machines. For ventilator manufacturers the introduction of decision support capabilities into ventilators is attractive: it may allow to optimize the therapeutic efficiency of their products, and it is technically feasible due to the power of the embedded chips. Figure 7 shows the possible architecture for a smart ventilator. This machine integrates all data processing steps: i) to give to the clinician a synthetic view of the patient's state evolution, through an easy to use interface; ii) and to operate automatically some parts of mechanical ventilation process, such as the weaning. This machine is connected to several monitors (cardiac frequency monitor, pulse oxymeter, ...) and to the hospital network for additional data (such as blood gas measurements). Above a realtime kernel for the tactical component functions (i.e. data acquisition, data processing and ventilation mode generation), via the standard components of the ventilator (i.e. electronics sensors and pneumatic actuators), the strategic layer is decomposed in several interconnected layers. For purposes of maintenance and reuse of knowledge bases developed and validated in several medical centres, a specific expert interface is available. 


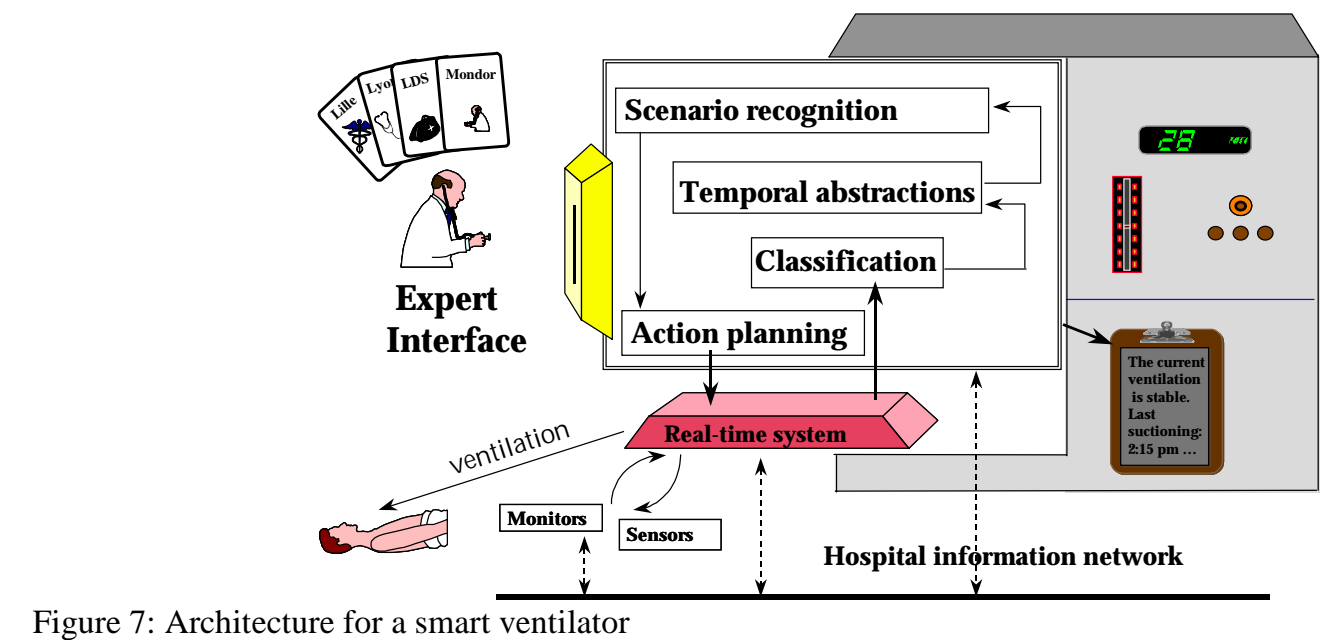

Figure 7: Architecture for a smart ventilator

A simple interface synthesizes the ventilation and therapy evolution. Automatic adjustment of ventilator settings is performed. The device integrates additional information via external monitors or the hospital network. Inside the ventilator, a specific architecture combines real-time functionnalities, such as data acquisition or ventilation modes generation, and knowledge-based functionnalities. An expert interface allows the maintenance and exchange of medical knowledge incorporated into the device.

Specific lung function testing manoeuvres could be automatically performed by the smart ventilator in order to refine the evaluation of the patient's state, and then the therapy. This information could be used to manage several ventilatory modes. Improvement of planning capacities, via the automatic recognition of high level clinical scenarios as they are developing, is a prerequisite to improve the predictions and the dynamic adaptation of the strategy. Interaction with the clinician could contribute to a dynamic adaptation of the strategy depending on information that cannot be directly accessible for the machine. Ventilatory care should be adapted to the patient's needs. Information provided directly by the patient about the quality of the assistance received could be incorporated into our future smart ventilators. 
Acknowledgements

The authors are indebted to colleagues of the INSERM U492 laboratory and of Intensive Care Unit of Henri Mondor's Hospital for their collaboration. We wish to thank Professor Alain Harf for his stimulating advice and thoughtful contributions.

\section{References}

1 Aiping J: The design and development of a knowledge-base ventilatory and respiratory monitoring system. PhD thesis, Univ. of Nashville, (TE), 1991.

${ }^{2}$ Brochard L, Rauss A, Benito S, et al: Comparison of three methods of gradual withdrawal from ventilatory support during weaning from mechanical ventilation. Am J Respir Crit Care Med 150: 896-903, 1994.

${ }^{3}$ Brünner J: Principles and history of closed-loop control mechanical ventilation. in this issue.

${ }^{4}$ Calvelo D, Chambrin M, Pomorski D, et al: Local trend extraction and visualisation for ICU monitoring. Artif Intell in Med to appear, 2000.

${ }^{5}$ Chambrin M-C, Chopin C, and Mangalaboyi KH: Autoregulated inspiratory support system. In 14th IEEE-EMBS Conference, Paris, 1992, p 2419-2420.

${ }^{6}$ Chambrin MC, Ravaux P, Chopin C, et al: Computer-assisted evaluation of respiratory data in ventilated critically ill patients. Int J Clin Monit Comput 6: 211-215, 1989.

${ }^{7}$ Cimino J: Distributed cognition and knowledge-based controlled medical terminologies. Artif Intell in Med 12: 153-168, 1998.

${ }^{8}$ Dawant B, Uckun S, Manders E, et al: The SIMON project: model-based signal analysis and interpretation in intelligent patient monitoring. IEEE Trans Med Biol 12: 82-91, 1993. 
${ }^{9}$ Dean TL, and Wellman MP: Planning and Control. San Mateo CA, Morgan Kaufmann, 1991.

${ }^{10}$ Dojat M, Brochard L, Lemaire F, et al: A knowledge-based system for assisted ventilation of patients in intensive care units. Int J Clin Monit Comput 9: 239-250, 1992.

${ }^{11}$ Dojat M, Harf A, Touchard D, et al: Evaluation of a knowledge-based system providing ventilatory management and decision for extubation. Am J Respir Crit Care Med 153: 997-1004, 1996.

12 Dojat M, Miksch S, and Hunter J: Special Issue on: Knowledge-Based Information Management in Intensive Care and Anaesthesia. Artif Intell in Med (to appear), 2000.

${ }^{13}$ Dojat M, and Pachet F: Effective domain-dependent reuse in medical knowledge bases. Comput Biomed Res 28: 403-432, 1995.

${ }^{14}$ Dojat M, Pachet F, Guessoum Z, et al: NeoGanesh: A Working System for the Automated Control of Assisted ventilation in ICUs. Artif Intell in Med 11: 97-117, 1997.

15 Dojat M, Ramaux N, and Fontaine D: Scenario Recognition for Temporal Reasoning in Medical Domains. Artif Intell in Med 14: 139-155, 1998.

16 Dojat M, and Sayettat C: A realistic model for temporal reasoning in real-time patient monitoring. AAI 10: 121-143, 1996.

${ }^{17}$ East TD, K HL, Bradshaw RL, et al: Efficacy of computerized decision support for mechanical ventilation: results of a prospective multi-center randomized trial. In AMIA'99 Conference, Washington DC, 1999, p 203-217.

${ }^{18}$ East TD, Wallace CJ, Moris AH, et al: Computers in critical care. Critical care nursing clinics of north america 7: 203-217, 1995.

${ }^{19}$ Esteban A, and Alía I: Clinical management of weaning from mechanical ventilation. Intensive Care Med 24: 999-1008, 1998. 
${ }^{20}$ Fagan L: Representing time dependant relations in a medical settings. PhD thesis, Univ. of Standford (Ca), 1980.

${ }^{21}$ Guessoum Z, and Dojat M, A real-time agent model in an asynchronous object environment. In Van de Velde W, Perram JW (eds): Agents Breaking Away. Berlin, Springer, 1996, p 190-203.

${ }^{22}$ Haimowitz IJ, and Kohane IS: Managing temporal worlds for medical trend diagnosis. Artif Intell in Med 8: 299-324, 1996.

${ }^{23}$ Hayes-Roth B, Washington R, Ash D, et al: Guardian: a prototype intelligent agent for intensive-care monitoring. Artif Intell in Med 4: 165-185, 1992.

${ }^{24}$ Horn W, Miksch S, Egghart G, et al: Effective data validation of high-frequency data: timepoint, time-interval, and trend-based methods. Comp Bio Med 27: 389-409, 1997.

${ }^{25}$ Hunter J: Decision support in the operating theatre and intensive care: A personal view. Artif Intell in Med 11: 93-96, 1997.

${ }^{26}$ Lanzola G, Gatti L, Falasconi S, et al: A framework for building cooperative software agents in medical applications. Artif Intell in Med 16: 223-249, 1999.

${ }^{27}$ Larsson J, Hayes-Roth B, Gaba D, et al: Evaluation of a medical diagnosis system using simulator test scenarios. Artif Intell in Med 11: 119-140, 1997.

${ }^{28}$ Laubscher TP, Heinrichs W, Weiler N, et al: An adaptive lung ventilation controller. IEEE Trans Bio Eng 41: 51-58, 1994.

${ }^{29}$ Miksch S, Horn W, Popow C, et al: Utilizing temporal data abstraction for validation and therapy planning for artificially ventilated newborn infants. Artif Intell in Med 8: 543-576, 1997.

${ }^{30}$ Miller P: Goal directed critiquing by computer: ventilator management. Comput Biomed Res 18: 422-438, 1985. 
${ }^{31}$ Morik K, Imhoff M, Brockhausen P, et al: Knowledge discovery and knowledge validation in intensive care. Artif Intell in Med to appear, 2000.

${ }^{32}$ Morris A: Computerized protocols and bedside decision support. Crit Care Clin 15: 523-545, 1999.

${ }^{33}$ Musen M: Modern architectures for intelligent systems: resuable ontologies and problemsolving methods. In AMIA Conference, Orlando, FL, 1998, p 46-52.

${ }^{34}$ Musen MA: Dimensions of knowledge sharing and reuse. Comput Biomed Res 25: 435-467, 1992.

${ }^{35}$ Nemoto T, Hatzakis G, Thorpe C, et al: Automatic control of pressure support mechanical ventilation using fuzzy logic. Am J Respir Crit Care Med 160: 550-556, 1999.

${ }^{36}$ Newell A: The knowledge level. Artif Intell 18: 87-102, 1982.

${ }^{37}$ Randolph A, Clemmer T, East T, et al: Evaluation of compliance with a computerized protocol: weaning from mechanical ventilator support using pressure support. Comput Methods Programs Biomed 57: 201-215, 1998.

${ }^{38}$ Rector A, Bechhofer S, Goble C, et al: The Grail concept modeling language for medical terminology. Artif Intell 9: 139-171, 1997.

${ }^{39}$ Rudowski R, Frostell C, and Gill H: A knowledge-based system for mechanical ventilation of the lungs. The KUSIVAR concept and prototype. Comp Biomed Res 30: 59-70, 1989.

${ }^{40}$ Rutledge GW, Thomsen GE, Farr BR, et al: The design and implementation of a ventilatormanagement advisor. Artif Intell in Med 5: 67-82, 1993.

${ }^{41}$ Schaüblin J, Derighetti M, Feigenwinter P, et al: Fuzzy control of mechanical ventilation during anesthesia. Brit J Anaest 77: 636-641, 1996. 
${ }^{42}$ Shahar Y: A framework for knowledge-based temporal abstraction. Artif Intell 90: 79-133, 1997.

${ }^{43}$ Shahar Y, Miksch S, and Johnson P: The Asgaard project: a task-specific framework for the application and critiquing of time-oriented clinical guidelines. Artif Intell in Med 14: 29-51, 1998.

${ }^{44}$ Sittig D: A computerized patient advice system to direct ventilatory care. PhD thesis, Univ. of Salt lake city (UT), 1988.

${ }^{45}$ Steimann F: The intepretation of time-varying data with DiaMon-1. Artif Intell in Med 8: 343$355,1996$.

${ }^{46}$ Strickland JH, and Hasson JH: A computer-controlled ventilator weaning system. Chest 100: 1096-1099, 1991.

${ }^{47}$ Strickland JH, and Hasson JH: A computer-controlled ventilator weaning system. Chest 103: 1220-1226, 1993.

${ }^{48}$ Tsien CL, Kohane IS, and McIntosh N: Multiple signal integration by decision tree induction to detect false alarms in the intensive care unit. Artif Intell in Med to appear, 2000.

49 Vilhelm C: Think!: A unified numerical-symbolic knowledge representation scheme and reasoning system. Artif Intell 116: 67-85, 2000.

${ }^{50}$ Younes M: Proportional Assist Ventilation, a new approach to ventilatory support. Am. Res. Respir. Dis. 145: 114-120, 1992.

${ }^{51}$ Zilberstein S, and Russell S: Optimal composition of real-time systems. Artif Intell 82: 181213, 1996. 